\title{
Descripción de una población de pacientes portadores de enfermedad pulmonar intersticial asistidos en el Hospital Pasteur de Montevideo, Uruguay.
}

\author{
Description of a population of patients bearing interstitial lung disease \\ assisted al Hospital Pasteur in Montevideo, Uruguay. \\ Descrição de uma população de pacientes com doença pulmonar \\ intersticial atendidos no Hospital Pasteur em Montevidéu, Uruguai.
}

Javier Gaudiano

ORCID: 0000-0003-3824-4695 Internista. Asistente Clínica Médica.

UdelaR.

Sofía de Betolaza

ORCID: 0000-0001-6843-2633 Internista. Asistente Clínica Médica.

Mauricio Amara ORCID: 0000-0002-5479-1130 Internista. Neumólogo. Profeso Adjunto Clínica Médica. UdelaR.
Resumen: Introducción: Las enfermedades pulmonares intersticiales son un grupo muy heterogéneo de afecciones que tienen manifestaciones clínicas, radiológicas y funcionales en común. Su epidemiología está documentada en forma parcial y su etiología es muy variada. El diagnóstico se basa en la clínica, la tomografía computada de alta resolución y, en algunos casos, la histología. Objetivos: describir la población de pacientes con diagnóstico de enfermedad pulmonar intersticial asistidos en la Policlínica de Intersticiopatías del Hospital Pasteur en el período comprendido entre el 5 de mayo de 2017 y el 17 de mayo de 2019. Materiales y métodos: Estudio descriptivo, observacional y transversal. Se utilizó un muestreo por conveniencia. Las variables analizados fueron: edad, sexo, comorbilidades, etiología, patrón tomográfico predominante, funcional respiratorio (Test de Marcha 6 Minutos y espirometría forzada con difusión de monóxido) y tratamiento instituido. Se obtuvo consentimiento informado y el trabajo fue aprobado por el Comité de Bioética del Hospital Pasteur. Resultados: 20 de los 42 pacientes asistidos eran portadores de enfermedad pulmonar intersticial. Predominó el sexo femenino (14 pacientes, $70 \%$ ). La edad media fue $60 \pm 16.84$ años. La comorbilidad más frecuente fue el tabaquismo (13 pacientes, $65 \%)$. En 6 pacientes (30\%) se identificaron exposiciones ambientales o laborales de riesgo. La etiología fue determinada en 15 pacientes (75\%), siendo en 10 pacientes (50\%) asociada a enfermedades autoinmunes sistémicas y en 3 pacientes (15\%) neumonitis por hipersensibilidad. La mayoría (11 pacientes, 55\%) tuvieron patrones tomográficos inespecíficos. $94.11 \%$ tuvieron un patrón restrictivo en el estudio funcional respiratorio, predominando la restricción moderada a severa. La difusión monóxido media fue $47.25 \pm 16.71 \% .11$ pacientes $(55 \%)$ fueron tratados con corticoides sistémicos y 2 pacientes necesitaron oxígeno a permanencia. Conclusiones: Las características demográficas, etiología, patrones tomográficos y tratamiento instituidos están influenciados por un sesgo de selección, ya que una elevada proporción de pacientes son derivados desde policlínicas de enfermedades autoinmunes sistémicas. Es fundamental un manejo integral de estos pacientes porque la asociación con otras enfermedades que pueden contribuir en la sintomatología es frecuente. Las enfermedades pulmonares intersticiales encontradas son mayormente severas y de diagnóstico tardío.

Palabras clave: enfermedad pulmonar intersticial, tomografía computada de alta resolución, funcional respiratorio.

Abstract: Introduction: Interstitial lung diseases are a very heterogeneous group of conditions that have common clinical, radiological and functional manifestations. Its epidemiology is partially documented and its etiology is very varied. The diagnosis is based on the clinic, high resolution computed tomography and, in some cases, histology. Objectives: to describe the population of 
patients diagnosed with interstitial lung disease assisted at the Hospital Pasteur Polyclinic for Interstitiopathies in the period between May 5, 2017 and May 17, 2019. Materials and methods: Descriptive, observational and cross. Convenience sampling was used. The variables analyzed were: age, sex, comorbidities, etiology, predominant tomographic pattern, respiratory function (6-minute walk test and forced spirometry with monoxide diffusion) and instituted treatment. Informed consent was obtained and the work was approved by the Bioethics Committee of the Pasteur Hospital. Results: 20 of the 42 assisted patients were carriers of interstitial lung disease. Female sex predominated (14 patients, $70 \%)$. The mean age was $60 \pm 16.84$ years. The most frequent comorbidity was smoking (13 patients, $65 \%$ ). Environmental or occupational risk exposures were identified in 6 patients (30\%). The etiology was determined in 15 patients (75\%), being associated with systemic autoimmune diseases in 10 patients $(50 \%)$ and hypersensitivity pneumonitis in 3 patients (15\%). Most (11 patients, 55\%) had nonspecific tomographic patterns. $94.11 \%$ had a restrictive pattern in the functional respiratory study, with moderate to severe restriction prevailing. The average monoxide diffusion was $47.25 \pm 16.71 \%$. 11 patients (55\%) were treated with systemic corticosteroids and 2 patients required oxygen permanently. Conclusions: The demographic characteristics, etiology, tomographic patterns and instituted treatment are influenced by a selection bias, since a high proportion of patients are derived from polyclinics for systemic autoimmune diseases. Comprehensive management of these patients is essential because the association with other diseases that may contribute to the symptoms is frequent. The interstitial lung diseases found are mostly severe and late diagnosed.

\section{Keywords: Interstitial Lung Disease, high resolution computed tomography, spirometry}

Resumo: Introdução: As doenças pulmonares intersticiais são um grupo muito heterogêneo de condições que apresentam manifestações clínicas, radiológicas e funcionais comuns. Sua epidemiologia está parcialmente documentada e sua etiologia é muito variada. O diagnóstico é baseado na clínica, na tomografia computadorizada de alta resolução e, em alguns casos, na histologia. Objetivos: descrever a população de pacientes com diagnóstico de doença pulmonar intersticial atendidos no Hospital Pasteur Polyclinic for Interstitiopathies no período de 5 de maio de 2017 a 17 de maio de 2019. Materiais e métodos: descritivos, observacionais e Cruz. Amostragem por conveniência foi utilizada. As variáveis analisadas foram: idade, sexo, comorbidades, etiologia, padrão tomográfico predominante, função respiratória (teste de caminhada de 6 minutos e espirometria forçada com difusão de monóxido) e tratamento instituído. O consentimento informado foi obtido e o trabalho foi aprovado pelo Comitê de Bioética do Hospital Pasteur. Resultados: 20 dos 42 pacientes assistidos eram portadores de doença intersticial pulmonar. O sexo feminino predominou (14 pacientes, $70 \%$ ). A idade média foi de $60 \pm$ 16,84 anos. A comorbidade mais frequente foi o tabagismo (13 pacientes, $65 \%$ ). As exposições a riscos ambientais ou ocupacionais foram identificadas em 6 pacientes (30\%). A etiologia foi determinada em 15 pacientes (75\%), sendo associada a doenças autoimunes sistêmicas em 10 pacientes $(50 \%)$ e pneumonite por hipersensibilidade em 3 pacientes (15\%). A maioria (11 pacientes, 55\%) apresentava padrões tomográficos inespecíficos. 94,11\% apresentaram padrão restritivo no estudo respiratório funcional, prevalecendo restrição moderada a grave. A difusão média de monóxido foi de $47,25 \pm 16,71 \%$. 11 pacientes $(55 \%)$ foram tratados com corticosteróides sistêmicos e 2 pacientes necessitaram de oxigênio permanentemente. Conclusões: As características demográficas, etiologia, padrões tomográficos e tratamento instituído são influenciados por um viés de seleção, pois uma alta proporção de pacientes é derivada de policlínica para doenças autoimunes sistêmicas. O manejo abrangente desses pacientes é essencial, pois a associação com outras doenças que podem contribuir para os sintomas é frequente. As doenças pulmonares intersticiais encontradas são na maioria graves e diagnosticadas tardiamente.

Palavras-chave: doença pulmonar intersticial (IPE), tomografia computadorizada de alta resolução (TCAR), função respiratória 


\section{Introducción}

Las enfermedades pulmonares intersticiales (EPI) son un grupo muy heterogéneo de afecciones que tienen manifestaciones clínicas, radiológicas y funcionales comunes. ${ }^{1}$

La epidemiologia de las EPI está documentada en forma parcial, siendo su prevalencia e incidencia variables según la clasificación utilizada y según el origen de los datos, debido a que la mayoría de los estudios provienen de servicios de neumología, introduciendo importantes sesgos de selección. Aun así, existe común acuerdo en que las EPI más frecuentes son la fibrosis pulmonar idiopática (FPI) y la sarcoidosis, seguidas por las neumonitis por hipersensibilidad y las asociadas a enfermedades del colágeno.

La etiología de las EPI es muy variada. En la actualidad se conocen más de 150 causas diferentes, aunque sólo en aproximadamente el $35 \%$ de ellas es posible identificar el agente causal. Se clasifican en tres grupos: neumonías intersticiales idiopáticas, las de causa conocida o asociadas a otras entidades clínicas bien definidas y las primarias o asociadas a otras enfermedades no bien definidas.

El primer grupo está constituido por entidades definidas histológicamente dentro de las cuales se destaca por su frecuencia la FPI. En el segundo grupo se incluyen manifestaciones pulmonares de las enfermedades del colágeno que muchas veces tienen histología indistinguible de las primeras. Asimismo, las vinculadas a exposición a polvos inorgánicos (neumoconiosis), inducidas por fármacos y radioterapia, así como las producidas por exposición a polvos orgánicos (neumonitis por hipersensibilidad) se encuentran dentro de este grupo. El tercer grupo está ejemplificado por la sarcoidosis y eosinofilias pulmonares. ${ }^{2}$

Además de la etiología, los problemas más relevantes que se plantean en la práctica clínica son el diagnóstico precoz, el tratamiento y la identificación de factores predictivos para valorar su evolución. ${ }^{3}$

El síntoma cardinal es la disnea, por lo general lentamente progresiva, seguido por la tos seca. Al examen físico los hallazgos son variables, siendo significativos los estertores crepitantes gruesos ("velcro") y el hipocratismo digital. ${ }^{2}$

La tomografía computada de alta resolución (TCAR) es el estudio de mayor jerarquía en las EPI ya que permite la confirmación del diagnóstico, así como la valoración de la extensión de lesiones parenquimatosas y su naturaleza. En un porcentaje importante de casos, una historia clínica compatible, asociada a un patrón tomográfico típico permitirá establecer el diagnostico etiológico prescindiendo del estudio anatomopatológico. ${ }^{2,4}$

El funcional respiratorio es un elemento indispensable para valorar la repercusión funcional en las EPI, teniendo valor pronóstico. Es por lo tanto útil en el control evolutivo y en la valoración de la respuesta al tratamiento. En un $15 \%$ de las EPI, la alteración del funcional respiratorio puede ser la primera manifestación de la enfermedad. El patrón restrictivo en la espirometría forzada es la alteración característica. La capacidad de transferencia pulmonar del monóxido de carbono (DLCO) esta disminuida, siendo éste el indicador más sensible., ${ }^{2,5,6}$

El test de marcha de 6 minutos (TM6M) es un elemento útil a la hora de valorar repercusión funcional y evolución de la enfermedad dada su fácil aplicación y disponibilidad. ${ }^{6}$

El diagnóstico definitivo y específico de las EPI requerirá en algunos casos el estudio histológico del parénquima pulmonar, ya sea mediante criobiopsia o biopsia pulmonar quirúrgica. ${ }^{7}$

Los pilares del tratamiento consisten en evitar la exposición al agente causal (en los casos en que aplica), suprimir el componente inflamatorio de la enfermedad en los casos en que existe (mediante el uso de glucocorticoides, inmunosupresores o terapias biológicas) y tratar las principales complicaciones (insuficiencia respiratoria e infecciones). Más recientemente se ha extendido el uso de fármacos antifibróticos (específicamente para FPI) lo cual ha desatado una verdadera revolución a nivel académico y terapéutico, surgiendo sucesivamente otras indicaciones de éstos fármacos para otras enfermedades intersticiales fibrosantes, a medida que nuevos ensayos clínicos van demostrando resultados favorables. ${ }^{7}$

El Hospital Pasteur es un centro hospitalario de tercer nivel perteneciente a la Administración de Servicios de Salud del Estado (ASSE), que asiste a una importante proporción de pacientes de Salud Publica contando también con un rol académico en la formación tanto de pregrados como de posgrados. En mayo de 2017 comenzó a funcionar la Policlínica de Intersticiopatías del Hospital Pasteur como parte de la Clínica Médica 2, constituida por un equipo que incluye 
posgrados y residentes de medicina, docentes, internistas y un neumólogo con la meta de captar y optimizar la atención y el seguimiento de estos pacientes.

El objetivo general de este trabajo es describir la población de pacientes asistidos en la Policlínica de Intersticiopatías del Hospital Pasteur. Los objetivos específicos son determinar la procedencia de los pacientes, sus características demográficas y comorbilidades así como la etiología, patrón tomográfico, patrón funcional y tratamiento de su enfermedad.

\section{Material y métodos}

Se trata de un estudio descriptivo, observacional y transversal que incluye a los 42 pacientes asistidos en la Policlínica de Intersticiopatías del Hospital Pasteur en el período comprendido entre el 5 mayo del 2017 hasta 17 de mayo del 2019. Se utilizó un muestreo por conveniencia.

Los pacientes que concurrieron a la policlínica en dicho período son aquellos con clínica y/o imagenología compatibles derivados desde la internación en sala de Medicina, policlínica general o de especialidades, servicio de emergencia y desde el interior del país.

Del total de pacientes que concurrieron a la policlínica, se determinó cuáles eran portadores de EPI definida tomando en cuenta: clínica compatible, factores de riesgo ambientales o enfermedades predisponentes, pruebas funcionales y sobre todo la imagenología compatible, a través de la TCAR de tórax. De estos pacientes se realizó una descripción exhaustiva mediante la revisión de sus historias clínicas que incluyó las siguientes variables: edad, sexo, comorbilidades, etiología, patrón tomográfico predominante, pruebas funcionales respiratorias (TM6M y espirometría forzada con DLCO) y tratamiento instituido.

La severidad del patrón restrictivo en el funcional respiratorio se determinó mediante el valor obtenido de Capacidad Vital Forzada (CVF) post administración de broncodilatador (porcentaje del valor teórico esperado), según lo establecido en ATS5, de manera que se consideró alteración restrictiva leve si CVF $\geq 65 \%$ y $<$ LIN (límite inferior normal), alteración restrictiva moderada si CVF $<65 \%$ y $\geq 50 \%$ y alteración restrictiva avanzada si CVF $<50 \%$.

La severidad del descenso de la DLCO obtenido (expresado mediante el porcentaje del valor teórico esperado) se determinó siguiendo la normativa de ATS5 según la cual el descenso es leve si es $\geq 60 \%$ y $<$ LIN (límite inferior normal), moderado si $\geq 40 \%$ y $<60 \%$ y severa si es $<$ $40 \%$.

El TM6M se consideró alterado cuando la distancia recorrida por el paciente fue menor al límite inferior esperado.

Las variables cualitativas fueron expresadas mediante frecuencias absolutas y frecuencias relativas porcentuales. Las variables cuantitativas fueron expresadas mediante la media y el desvío estándar.

El procesamiento de los datos se realizó en el programa EPI-DATA versión 3.1 y PSPP.

Los pacientes firmaron un consentimiento informado diseñado específicamente para este trabajo. El protocolo de investigación fue aprobado por el Comité de Bioética del Hospital Pasteur.

\section{Resultados}

En el período estudiado se asistieron en la policlínica de Intersticiopatías del Hospital Pasteur 42 pacientes en total, de los cuales 20 eran portadores de EPI definida según los criterios ya detallados. Entre los principales diagnósticos diferenciales que presentaron los 22 pacientes restantes se destacan: Enfermedad Pulmonar Obstructiva Crónica (EPOC), asma, hipertensión pulmonar y enfermedad bronquiectásica.

De los 20 pacientes con diagnóstico de EPI, 19 eran procedentes del Hospital Pasteur; 12 fueron derivados a la policlínica tras el alta de internación en sala de medicina o tras consultar en el servicio de emergencia y 7 fueron derivados desde otras policlínicas (6 de estos pacientes fueron derivados desde Policlínica de Enfermedades Autoinmunes Sistémicas (EAS)). Solamente un paciente portador de EPI no era procedente del Hospital Pasteur. 
Entre los pacientes portadores de EPI, 14 (70\%) eran de sexo femenino y $6(30 \%)$ eran de sexo masculino. La edad promedio fue de $60 \pm 16.84$ años, siendo la edad mínima 21 años y la edad máxima 83 años.

La comorbilidad más prevalente fue el tabaquismo, de los cuales 6 eran tabaquistas activos y 7 eran ex tabaquistas. Le siguió en frecuencia la enfermedad por reflujo gastroesofágico (ERGE), EPOC, síndrome de apnea-hipopnea obstructiva del sueño (SAHOS), asma, enfermedad bronquiectásica y cardiopatía isquémica (Gráfica 1). Hubo pacientes que presentaron más de una comorbilidad.

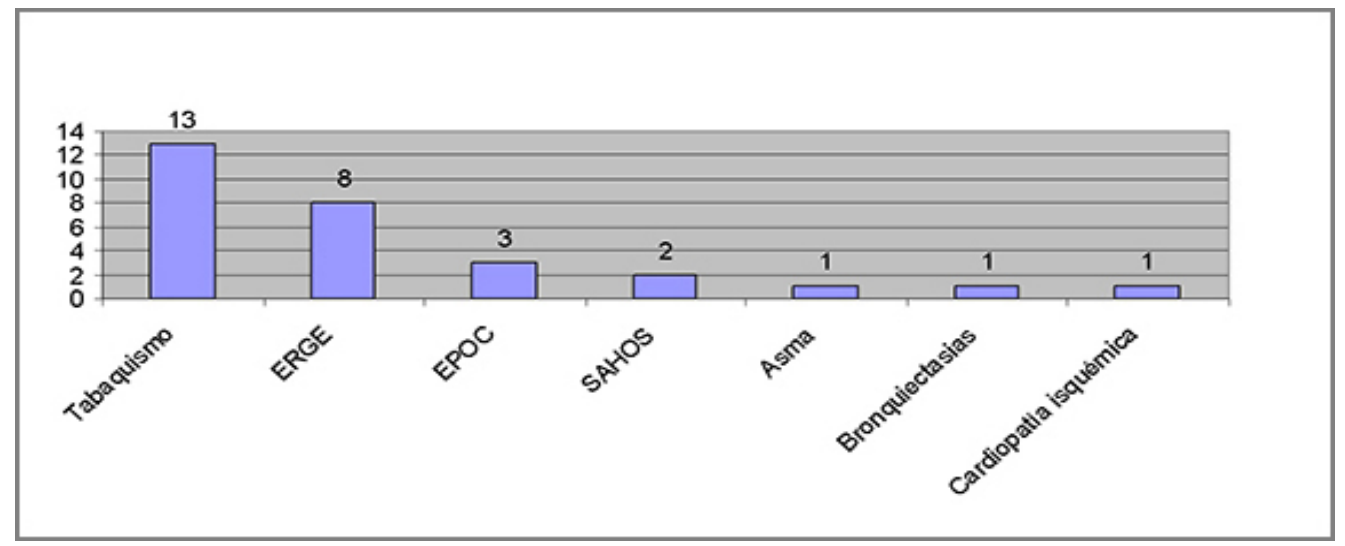

En 6 pacientes (30\%) se identificaron exposiciones de riesgo; 4 eran exposiciones ambientales y 2 eran laborales.

La etiología de la EPI fue determinada en 15 pacientes (75\%). 4 pacientes no tenían completada su valoración al momento de realizar este estudio, mientras que un paciente no tenía etiología definida de su EPI tras completar la valoración diagnóstica. Este paciente en particular no pudo realizarse una biopsia pulmonar debido a la severa repercusión funcional al momento del diagnóstico, lo cual contraindicaba el procedimiento.

En 10 pacientes (50\%) la EPI fue secundaria a una EAS correspondiendo a artritis reumatoide $(A R)$ en 2 pacientes, esclerosis sistémica (ES) en 2 pacientes, enfermedad mixta del tejido conectivo (EMTC) en un paciente, síndrome antisintetasa en un paciente, solapamiento de lupus eritematoso sistémico (LES) con EMTC en un paciente, Síndrome de Sjögren en un paciente, Granulomatosis con Poliangeítis (Ex -Granulomatosis de Wegener) en un paciente y Enfermedad Indiferenciada del Tejido Conjuntivo en un paciente. En 3 pacientes se hizo diagnóstico de neumonitis por hipersensibilidad; en un paciente fue secundaria a la exposición a aves, en otro fue secundaria a la fabricación de jabones y en un paciente que trabajaba como envasador de especies, la neumonitis se consideró secundaria a la exposición a las mismas. En un paciente se atribuyó la EPI a neumotoxicidad por el quimioterápico Capecitabine. Un paciente era portador de una eosinofilia pulmonar, a forma de neumonia eosinofílica crónica (Gráfica 2).

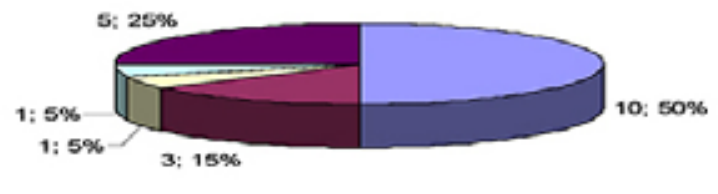

\section{OEAIS}

a Noumonitis por hiporsensibilidad a Toxicidad por quimioterápice

O Eosinoflia pulmonar

- Indeterminado

En cuanto a los patrones tomográficos de los pacientes portadores de EPI, se encontró que $6(30 \%)$ eran compatibles con Neumonitis Intersticial No Específica (NINE), 3 eran compatibles con Neumonía Intersticial Usual (NIU) y los 11 restantes no tenían un patrón tomográfico definido, sino afectación intersticial de forma y entidad variable.

17 de los 20 pacientes con diagnóstico de EPI tenían estudio funcional respiratorio (EFR) realizado a la fecha, mostrando un franco predominio del patrón restrictivo (16 pacientes, $94.11 \%)$. Un paciente tenía un EFR dentro de valores normales. De los 16 pacientes con un patrón 
restrictivo, 5 presentaron restricción leve, 5 presentaron restricción moderada y 4 presentaron restricción severa. En 2 pacientes no se registró la severidad de la restricción (Gráfica 3).

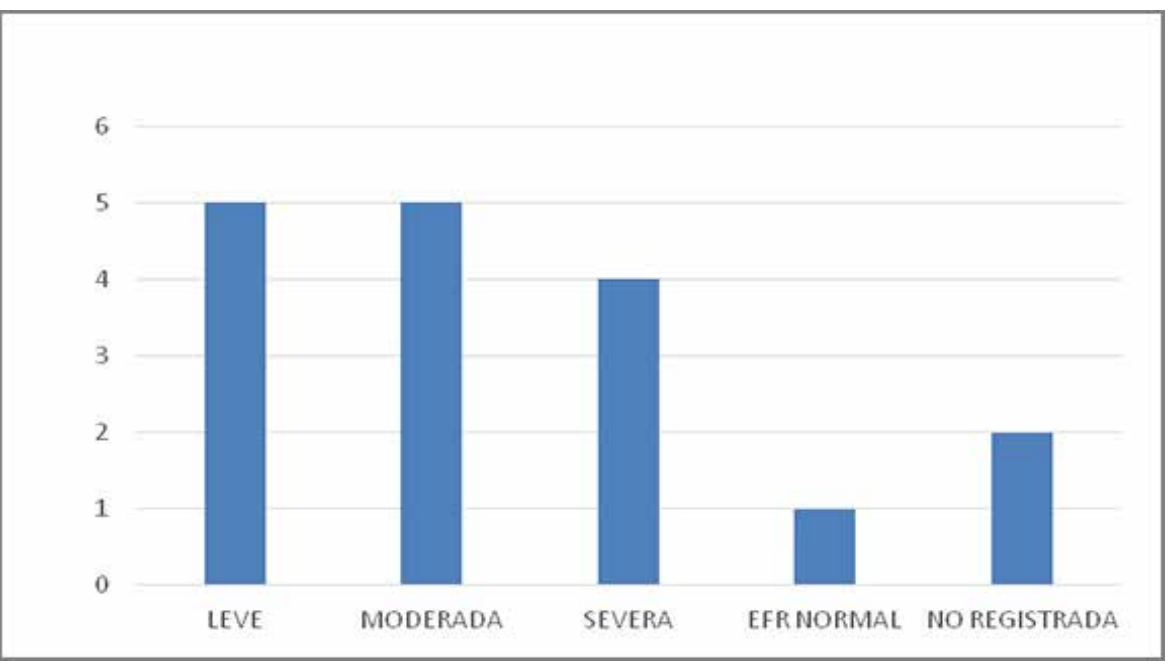

En 13 pacientes portadores de EPI se determinó la DLCO, siendo $47.25 \% \pm 16.71 \%$ el valor promedio. Su descenso fue leve en 2 pacientes, moderado en 5 pacientes y severo en 5 pacientes. Un paciente tuvo un valor de DLCO normal (Gráfica 4).

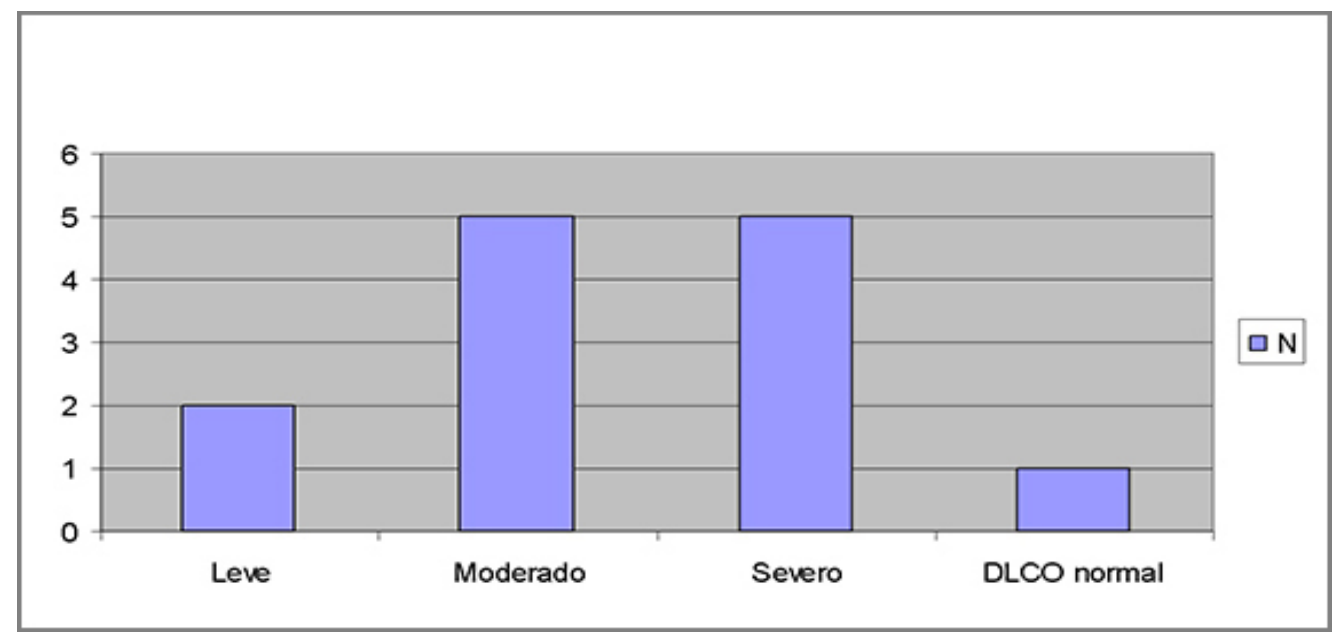

El TM6M fue llevado a cabo por 12 de los 20 pacientes portadores de EPI, encontrándose alterado en 8 casos (66.66\%).

Si se analiza el tratamiento instituido a los pacientes portadores de EPI, $11(55 \%)$ de los 20 pacientes recibían tratamiento con corticoides sistémicos al momento de realizar este estudio, 9 pacientes $(45 \%)$ recibían tratamiento con inmunosupresores y 2 pacientes $(10 \%)$ recibían tratamiento con fármacos biológicos (Rituximab). 6 pacientes $(30 \%)$ recibían tratamiento con corticoides sistémicos asociados a inmunosupresores. 2 pacientes tenían oxígeno a permanencia y un paciente fue conectado con el servicio de Cuidados Paliativos.

\section{Discusión}

El presente estudio analiza diferentes aspectos de 20 pacientes portadores de EPI que fueron valorados en la policlínica de Intersticiopatías del Hospital Pasteur en sus primeros dos años de funcionamiento. Se trata de un número pequeño pero considerable, teniendo en cuenta la relativamente baja prevalencia de estas enfermedades, la frecuencia bimensual de las consultas y que la policlínica es un servicio nuevo, que comenzó a funcionar en 2017. Se destaca la existencia de un sesgo de selección en la muestra estudiada, ya que una importante proporción de los pacientes vistos en la policlínica son derivados desde policlínicas de EAS del Hospital, lo cual explica el alto porcentaje de EPI asociada a EAS que se asisten. 
De las características demográficas de los pacientes con EPI se destaca la prevalencia del sexo femenino (70\%), que puede explicarse también por el alto porcentaje de pacientes derivados de policlínicas de EAS, cuyos pacientes en la mayor parte de los casos son mujeres. En un estudio multicéntrico realizado entre 2008 y 2017 que incluyó a 59 pacientes registrados en policlínicas de EAS y Neumología de centros públicos y privados de Montevideo con diagnóstico definido de EPI en el contexto de EAS, el $88 \%$ de los pacientes eran de sexo femenino. ${ }^{6}$

El amplio rango de edades de los pacientes con EPI denota la variedad de las etiologías y agentes ambientales causales.

La comorbilidad más prevalente fue el tabaquismo (65\%) seguida por la ERGE (40\%); esta última podría ser responsable de algunos síntomas respiratorios como la disnea o la tos y podría contribuir a la afectación del intersticio pulmonar. Luego surgen una serie de enfermedades de la esfera respiratoria entre las cuales se incluyen la EPOC, el asma, el SAHOS y la enfermedad bronquiectásica, así como la asociación con cardiopatía, que seguramente contribuyen en la génesis de los síntomas y permiten inferir que la disnea de la mayor parte de los pacientes puede ser de causa multifactorial. El tratamiento integral de estas comorbilidades es fundamental para lograr una mejoría sintomática subjetiva.

Las exposiciones a inhalantes ambientales o laborales estuvieron presentes en un considerable número de casos (30\%). En algunos fueron consideradas como la causa principal de la EPI; en otros la EPI fue atribuida a otra causa de mayor jerarquía, aunque es probable que en todos contribuyan a la afectación pulmonar y a la presencia de síntomas. No es sorprendente considerando que la mayor parte de la población asistida tiene un mal medio socioeconómico y cultural, con alta prevalencia de actividades laborales en fábricas y escaso uso de medidas de protección. A esto se le suma la importante contaminación ambiental por exposición a biomasa y/o humos de fábricas cercanas a las viviendas a los que están expuestos muchos de los pacientes.

La etiología de las EPI fue determinada en un 75\%. Este porcentaje es elevado comparado con lo que está descrito a nivel internacional, estimándose que se logra determinar la etiología de las EPI solamente en un $35 \%$ de los casos, quedando el resto sin definir.2 Esto tiene varias explicaciones; por un lado está la alta frecuencia de EPI asociada a EAS en pacientes que ya concurrieron a la policlínica con el diagnóstico de su EAS, lo cual hacía más fácil el estudio etiológico. Por otro lado, hay una proporción significativa de EPI atribuida a neumonitis por hipersensibilidad, diagnóstico que surge en general directamente tras un interrogatorio completo. La considerable proporción de EPI que se consideró secundaria a EAS (50\%) puede explicarse por el sesgo de selección de la población asistida que ya fue descrito más arriba. Las EAS más prevalentes (AR y ES) fueron las que con más frecuencia se asocian con EPI según la literatura. En el estudio citado de Díaz, Consani, et al., la AR y la ES fueron las EAS que con mayor frecuencia se asociaron a EPI (28\% y $22 \%$ respectivamente del total de la muestra). 6 Llama la atención la ausencia de diagnóstico de FPI entre los pacientes asistidos, que es la EPI más prevalente según la bibliografía. ${ }^{2}$

El patrón tomográfico más frecuente (30\%) fue la NINE, lo cual no es llamativo dada la alta prevalencia de EPI asociada a EAS, siendo este el patrón que más se vincula con estas enfermedades. De manera similar, en el estudio citado de Díaz, Consani, et al., la prevalencia de NINE entre los patrones tomográficos fue del $64 \% .^{6}$

En el análisis de los EFR se destaca el predominio del patrón restrictivo en $94.11 \%$ que es el patrón espirométrico más característico de los pacientes portadores de intersticiopatías. La DLCO estaba descendida en la casi totalidad de los pacientes en los que se determinó, tratándose de otra de las alteraciones fundamentales que definen la presencia de una EPI. Se destaca la severidad de dicho descenso que fue moderado o severo en la mayoría de los casos, así como la severidad de la restricción en los funcionales respiratorios, que fue moderada o severa en un alto porcentaje de casos. Esto podría indicar un diagnóstico tardío, en fases avanzadas, de esta patología respiratoria que también puede vincularse con aspectos culturales (consultas tardías, minimización de la sintomatología, falta de adherencia a controles y realización de estudios). En contraposición, en el estudio de Díaz et al., la CVF y DLCO promedio fueron $80 \%$ y $71 \%$ respectivamente, reflejando una menor severidad de la afectación respiratoria al momento del diagnóstico, con una media de tiempo de un año entre el comienzo de los síntomas y el diagnóstico. ${ }^{7}$ Se destaca la importancia de siempre tener un alto índice de sospecha para no pasar por alto estas enfermedades que muchas veces son de difícil diagnóstico, sobre todo en aquellos pacientes con factores de riesgo para las mismas (diagnóstico de EAS, exposiciones ambientales o laborales, antecedentes familiares, etc.). La alteración en el TM6M refleja una importante repercusión funcional en los pacientes portadores de EPI analizados. 
El tratamiento instituido en los pacientes estudiados está directamente asociado a las etiologías de las EPI determinadas. La alta prevalencia de EPI asociada a EAS y de neumonitis por hipersensibilidad justifica la elevada frecuencia de uso de corticoides sistémicos (55\%). El compromiso respiratorio da severidad y es un elemento de mal pronóstico en las EAS; de ahí que en pacientes portadores de EPI asociada a EAIS esté indicado el uso de inmunosupresores, teniendo una alta prevalencia en la muestra analizada (45\%). La necesidad de oxigenoterapia domiciliaria en dos pacientes y de cuidados paliativos en un paciente son otros elementos que reflejan la severidad del compromiso respiratorio. En este estudio no se indagó sobre otros tratamientos recibidos por los pacientes para el manejo de comorbilidades.

\section{Conclusiones}

Existe un sesgo de selección entre los pacientes que concurren a la Policlínica de Intersticiopatías del Hospital Pasteur, ya que muchos son derivados desde policlínicas de EAS; esto es determinante al analizar las características demográficas de nuestra muestra, la etiología de la EPI, los patrones tomográficos y el tratamiento instituido.

La asociación con otras enfermedades respiratorias o patologías que pueden contribuir en la génesis de los síntomas es muy frecuente, siendo de fundamental importancia el manejo integral de estos pacientes para lograr adecuadas respuestas terapéuticas.

Se trata de una muestra portadora de EPI severas y de diagnóstico relativamente tardío, siendo de gran importancia tener un alto índice de sospecha que permita realizar diagnósticos más precoces.

\section{Conflicto de interés}

Los autores declaran no tener conflictos de interés.

\section{Bibliografía}

1- Cuervo R, Palau JM. Enfermedad pulmonar intersticial. Med Clin (Barc). 2003;121(11):426-30

2- Xaubet A, Ancochea J, Blanquer R, Montero C, Morell F, Rodríguez Becerra E, et al. Diagnóstico y tratamiento de las enfermedades pulmonares intersticiales. Arch Bronco neumol. 2003;39(12):580-600

3- Xaubet A, Molina-Molina A, Sánchez M. Enfermedades pulmonares intersticiales difusas. Arch Bronco neumol. 2007;(43): 24-30

4- Ancochea J, Gómez J, Vilar J, Xaubet A. Consenso para el diagnóstico de las neumonías intersticiales idiopáticas. Arch Bronconeumol. 2010;46(Supl X): 2-21.

5- American Thoracic Society. Single -breath carbon monoxide diffusing capacity (Transfer factor) Recommendations for a standard technique- 1995 update. Am J Respir Crit Care Med. 1995; 152:2185-2198

6- Farreras C, Rozman. Compendio de Medicina Interna. Vol I. 6ª ed. Madrid: Elsevier; 2017.

7- Díaz C, Consani S, Torres V, Alonso F, Berez A. Estudio multicéntrico descriptivo de enfermedades pulmonares intersticiales asociadas a enfermedades autoinmunes en centros de salud en Montevideo, Uruguay. Rev. urug. med. interna. 2018; 2(3): 12-19.

\section{Aporte cada autor al trabajo}

Javier Gaudiano: recolección de datos, análisis e interpretación de los datos, análisis estadístico de los datos, redacción del manuscrito.

Sofía de Betolaza: recolección de datos, análisis e interpretación de los datos, análisis estadístico de los datos, redacción del manuscrito.

Mauricio Amaral: concepción y diseño del trabajo, revisión crítica del manuscrito. 